\title{
Research on the design and usability of Taiwan's native digital reminiscence therapy application system for patients with dementia
}

\author{
Pei-Fen Wu ${ }^{1}$, Wen-Fu Wang ${ }^{2}$, Kuang-Yi Fan ${ }^{3}$, Ya-Zhu Hsu' ${ }^{1}$ Chun-We Huang ${ }^{1}$ \\ ${ }^{1}$ Department of Information Management \& Master Program in Digital Content Technology and Management, National Changhua University of \\ Education, Changhua, Taiwan; ${ }^{2}$ Department of Neurology, Changhua Christian Hospital, Chunghua, Taiwan; ${ }^{3}$ The Graduate Institute of Animation \\ and Multimedia Design, National University of Tainan, Tainan, Taiwan \\ Contributions: (I) Conception and design: PF Wu, YZ Hsu, KY Fan; (II) Administrative support: PF Wu; (III) Provision of study material or patients: \\ All authors; (IV) Collection and assembly of data: YZ Hsu, CW Huang; (V) Data analysis and interpretation: PF Wu, YZ Hsu, KY Fan; (VI) \\ Manuscript writing: All authors; (VII) Final approval of manuscript: All authors. \\ Correspondence to: Professor Pei-Fen Wu. Department of Information Management, National Changhua University of Education, Changhua, Taiwan. \\ Email: pfwu@cc.ncue.edu.tw.
}

Background: We developed and designed a prototype of a digital reminiscence system featuring Taiwan's local representative reminiscence elements-memories for caregivers to accompany patients with dementia for reminiscence therapy. The goal of the study is to evaluate the applications of technologies to reminiscence therapy for person with dementia. The system was evaluated for usability and behavior observation from the perspective of patients with dementia, in order to put forward suggestions for the design of the system as a whole.

Methods: A total of 19 patients with moderate and low dementia who voluntarily participated in the study were recruited in Changhua area, including 4 males and 15 females with an average age of 76.8 years old. The experiment was carried out with one researcher accompanying one participant for the entire course. In the first stage of the experiment, participants were guided into the experimental situation by chatting about the reminiscence photos provided by participants. In the second stage, researchers assisted participants in system operation and explanation, and encouraged participants to tell their own experiences through the system, record their stories through reminiscence microphones, and share the process with relatives and friends after the activity was over. In the third stage, usability of system was evaluated by questionnaires, and qualitative analysis was carried out in cooperation with semi-structured, open interviews, and whole process audio visual records.

Results: This research developed an application system based on Taiwan's native reminiscence images, which can increase the interaction between participants and companions, and thus, make it easier for participants to immerse the implicit memory of themselves in the system through using. The results show that almost participants had highly accepted the presenting photos by computer, and had a good willing to try the operation of system. Through the digital reminiscence system, the reminiscence photos can be recorded and browsed quickly, and the native Taiwan materials and reminiscence atmosphere provided by this system are considered comfortable, allowing the patient with dementia to remind the fragments of their past life in the implicit memory.

Conclusions: This research developed an application system with Taiwan's reminiscence images as the theme, and discussed the benefits of usability for patients with dementia. Through observation and statistics, it is found that participants had high acceptance of new technologies. Through cognitive stimulation of implicit memory, the system would increase the interactions between the patience with dementia and caregivers. The process of the system is editable and recordable to retain the digital stories of patience with dementia. Finally, four influential factors were analyzed for the usability of the application system for patients with dementia: accessibility, usability, satisfaction, and learning. 
Keywords: Dementia; reminiscence therapy; cognitive stimulation therapy (CST); group therapy; usability

Received: 18 March 2019; Accepted:03 October 2019; Published: 28 November 2019.

doi: $10.21037 /$ ht.2019.10.01

View this article at: http://dx.doi.org/10.21037/ht.2019.10.01

\section{Introduction}

Dementia would cause mainly the disability and failure in the independence of life for the elderly around the world. It has caused serious impact on patients with dementia, caregivers, family members, communities, societies, and countries. Therefore, the Ministry of Health and Welfare of Executive Yuan in Taiwan actively promotes the prevention and care of dementia, shows concern for the personal, family, and social problems brought about by the cognitive dysfunction of dementia.

In order to delay aging speed and activate the brain, many scholars have devoted themselves to research the topic of dementia to delay the cognitive decline caused by dementia, pointing out that, in the aspect of cognitive and memory training for the elderly, cognitive function training or external memory aids to improve the forgettable behaviors (1). One of the most commonly used is cognitive stimulation therapy (CST), which is a non-drug intervention therapy that helps to improve cognitive function. It can be applied to the elderly with normal cognition and is suitable for patients with mild to moderate dementia $(2,3)$.

Reminiscence therapy, one of implicit memory therapy, is a therapeutic activity often used for the care of patients with dementia. It emphasizes that the process of memory is a pleasant experience and an important measure to maintain physical and mental health and quality of life. Reexperiencing familiar activities and reconnecting with past life events can promote communication to increase the socialization, produce both of self-confidence and pleasant effects (4-6). In the process of reminiscence therapy, patients with dementia can be encouraged to tell their own stories, share their past experiences, and learn to interact with society. Guillemot \& Urien (7) described 6 intrinsic motivations for narration among the elderly: (I) flattering the ego (self-confidence, achievement); (II) repairing the ego (to heal, resilience); (III) not being forgotten (transgenerational); (IV) sharing (education, learning); (V) to transmit (valorization, meaning to life); (VI) to testify (to remember, to communicate, to build resilience), whose point of view illustrates the psychological aspect of self-confidence, and also confirms the importance of the narrative process of the elderly in preventing and improving dementia.

Alaoui \& Lewkowicz (8) pointed out that science and technology can play an important role in increasing the ability of the elderly to participate in social interaction. The brain training game "Game Show", as developed by Savulich et al. (9), can improve the memory of patients with initial dementia and help patients to avoid some symptoms of cognitive deterioration. The use of assistive technology can provide cognitive or physical solutions for people with dementia, in order to improve the possibility of independent living for them (10-13).

Pecorini \& Duplaa (14) mentioned that combining narrative gerontology and digital storytelling to create "digital narrative gerontology" will bring a new promoter concept that will act positively on the "well-being" of the elderly, promote "aging-well", and share these positive values with the community. The elderly with dementia would have opportunities to participate in social interactions and take ownership of conversations when they access to rich and engaging multimedia reminiscence materials. The barriers would be reduced due to movement deficits during interactions with media (15). There are several reviews have been published on randomized control trials of reminiscence therapy interventions for both the general elderly (16) and the elderly with dementia (17-19). There is one review addressed systems that used information and communication technology (ICT) to deliver reminiscence therapy and concluded that using ICT is feasible for reminiscence therapy in a population with dementia (20). While most existing ICT systems are mainly based on cognitive function training.

This study introduces the reminiscence therapy process, one of implicit memory therapy, into a digital storytelling application system that the reminiscence materials based on the Taiwan's local life culture. That triggers the implicit memories of the elderly to achieve the effect of cognitive stimulation. This application not only considers patients with dementia, but also allows the caregivers to participate. The purposes of the study are: (I) to develop and design a prototype design of digital storytelling for a digital 

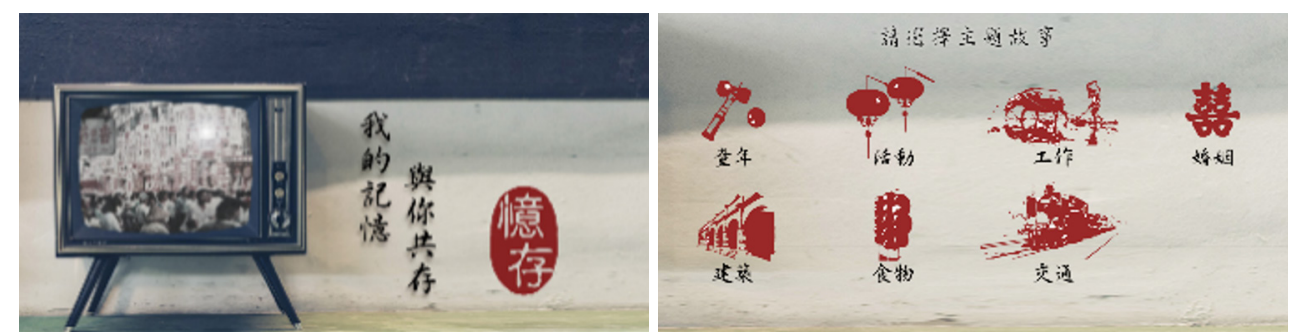

Figure 1 Interface design of the application system.

reminiscence system featuring Taiwan's representative reminiscence elements, in order to facilitate caregivers accompanying patients with dementia for reminiscence therapy, and (II) to explore the usability of this application system for patients with mild or moderate dementia.

\section{Methods}

The research framework was divided into two parts: one is system planning and design, the other is system evaluation and analysis of usability.

This study, developed and designed a prototype of a digital reminiscence system featuring Taiwan's local representative reminiscence elements-memories, provides caregiver with a system to accompany patients with dementia for reminiscence therapy. Old TV screen with nostalgic background songs guides users into a nostalgic atmosphere, as shown in Figure 1. The system, named as "RE-Stor" (means restory \& restore), takes "My memory, co-existed with you" as slogan. After selecting a theme, user can create their own story. The story creation of user can use the image material created by this system, or the personal photo album that uploaded by user. Recording the process of story creating, and merged with the photo to be a story video when process is finished.

In order to design program that is suitable for patients with dementia, the researcher actually participated organization of activities with the care institutions at the period of both planning and designing, and interviewed the caregivers for investigation. Simultaneously, the Taiwan's reminiscence elements were investigated through the questionnaire of Taiwan native reminiscence imagery, which was applied as the material for the system.

Reminiscence is conceptualized as a preference for common things in youth. The research subjects are all the elderly of Changhua natives. In order to establish the system material clearly to resonate with the subject, researchers went to Changhua reminiscence store and Lugang old street to find the raw material of reminiscence before the system was built. That would be convenience to cover all the nostalgic elements of different generations. A survey of nostalgic images in Taiwan was conducted and 185 questionnaires were collected. The nostalgic image survey is mainly based on the central Taiwan or the birthplace is in Changhua.

The nostalgic image questionnaire is divided into three parts. The first part investigates the representative characteristics of Taiwan natively; the second part sorts the nostalgic elements and constitute various themes. The final part is to finalize subject categories of the survey are divided into the following themes: childhood memories, food, clothing, building facilities, transportation/personalities, and education.

The system was evaluated by the usability and behavior observation from the perspective of patients with dementia. Preece et al. (21) considered that users can be observed the difference between in control environment and external activities. Through questionnaires and interviews, the user's need can be identified and serve as the basis for questionnaire design. Before the formal test, in order to examine the appropriateness of the experimental design process and questionnaire design, three subjects were recruited for pre-testing. The dementia status of the three subjects were an 80-year-old female without dementia, a 78-year-old with mild dementia, and an 83-year-old with moderate dementia. Through the behavioral observations of the three subjects, it was found that the elderly could not concentrate for a long time. Therefore, the experimental procedure of the formal test was revised according to the pre-test results, and the number of questions in the questionnaire was reduced from 20 to 11 by two experts and scholars. The content of questionnaire refers to the validated that is effective in detecting the interface in the pass research, and the questionnaires that has developed 
recently for measuring usability, such as WAMMI, PSSUQ, CSUQ, QUIS and SUS.

The original evaluation of system type, scale and reliability is modified by the suggestion of both two usability experts and two medical staff, to be suitable for this research. The survey facets are for efficiency, effectiveness, learning, memory and satisfaction, and also include basic personal data, surveys on literacy, education and the use of technology experience.

Under the guidance of researcher, the system operation was jointly carried out by caregivers (including family members) to use the digital reminiscence system to create stories of patients with dementia. The experiment involved 40 minutes of storytelling creation with recording. The researchers observed the behaviors and recorded the videos during the whole process. Finally, the person with dementia evaluated the usability of the system, which serves as reference for the design of the digital assistant system for dementia in the future.

The experimental sites were Lukang Christian Hospital and the dementia day care center in Changhua city. The subjects were determined, most of whom were diagnosed as dementia patients with CDR scores below 2, identified by Changhua Christian Hospital, and their families had a willing to participate. There were eight patients with mild dementia and 11 with moderate dementia, the youngest patient was 68 , the oldest was 85 , and the average age was 76.8. There were eight family members and two hospital volunteers participated.

In the first stage of the experiment, the researchers guided the subjects to recall patient's implicit memories by the old photos they carried to lead the subjects to enter the situation of reminiscence.

The second stage of the experiment, researchers gradually led the subjects into operation from the front page of the system to begin to enter reminiscence therapy officially.

In the third stage, the subjects were guided to select objects related to their own memories from the material library; recording and editing their digital stories while sharing their experiences.

In the fourth stage, the subjects reviewed and shared the stories that they had made.

In the fifth stage, the questionnaires were used to investigate the feelings of the patients with dementia when they used the system.

\section{Results}

This research developed an application system based on
Taiwan's native reminiscence images, which touch the implicit memory of patients with dementia to interact increasingly between participants and companions. The reminiscence images make it easier for participants to immerse themselves in the system. The results show that most participants had accepted highly the method of presenting photos by computer, and were willing to operate the system. Through the digital reminiscence system, old photos can be recorded and browsed more quickly, and the native Taiwan materials and reminiscence atmosphere provided by this system are considered comfortable, allow patients to relive many fragments of past life in their implicit memory.

\section{Discussion}

\section{Reminiscence therapy featuring Taiwan's image characteristics}

Through the investigation of reminiscence images of Taiwan, it can be found that, with Taiwan's social stability and economic growth, people with different ages has unique and obvious memory differences in people, things, and matters. For people over 70 years old, the selected reminiscence elements were relatively simple; on the contrary, the young would select the complicated. The system collected and classified reminiscence elements, and made them into the digital reminiscence application system. In addition to digitizing paper images and storing them in a database to manage files, it can also enable caregivers to choose materials closer to the user's past life as triggers the different patient for the process of reminiscence therapy.

\section{Degree of acceptance of patients with dementia for use of digital reminiscence application system}

Through the gradual guidance of the task, the rejection of patients with dementia to the new technology became relatively low, and timely encouragement from researchers and caregivers also improved the elderly's acceptance. The task design requires a simple introduction practice. It was the first time for all the patients with dementia to use 3C products; while some patients with dementia were not in the age of technological products, all they agreed to try to use after more encouragement and guidance. However, when they found they could smoothly drag the pictures on the screen and record their own voice, it increased their sense of accomplishment, and they were willing to further use the 
digital reminiscence application system.

As almost of the subjects in this experiment were literate, there was no obstacle to use the text interfaces in the system. The patients with dementia considered the reminiscence style of the system to be pleasing and comfortable pictures. As the materials conformed to their memories, thus, they were highly accepting and had willing to operate the technological method of presenting photos by computer. In the process of reminiscence, some subjects said that the current technology has brought them a great change in their life. Through the digital reminiscence system, they

Table 1 Factor analysis

\begin{tabular}{|c|c|c|c|c|}
\hline \multirow{2}{*}{ Factors } & \multicolumn{4}{|c|}{ Factor } \\
\hline & 1 & 2 & 3 & 4 \\
\hline Like interface & 0.879 & & & \\
\hline Appropriate material & 0.780 & & & \\
\hline Easy to learn and use & 0.774 & & & \\
\hline Rapid production & 0.639 & & & \\
\hline Need more explanation & 0.558 & & & \\
\hline Design is easy to understand & & 0.940 & & \\
\hline Comfortable to use & & 0.812 & & \\
\hline Imagination is consistent & & & 0.893 & \\
\hline Comfortable use & & & 0.827 & \\
\hline Overall satisfaction & & & 0.541 & \\
\hline Need help & & & & 0.872 \\
\hline
\end{tabular}

found that they could quickly browse through the photos. The materials made them feel comfortable in reminiscence atmosphere, which allowed them to relive many fragments of their past life from their implicit memory.

\section{Questionnaire reliability and validity evaluation}

The Cronbach's $\alpha$ value of the reliability test of the questionnaire is 0.803 , which shows that the research has high reliability. The validity Table 1 observes the results of the post-rotation component matrix, which shows that the usability questionnaire of this research has both of convergent validity and discriminatory validity.

\section{Factor analysis of the usability of the technology assistance system for patients with dementia}

According to literature, the questionnaire used in this study was divided into five major factors: efficiency, efficacy, learning, memory, and satisfaction. However, after using the SPSS analysis the principal component, only four factors were extracted. Figure 2 shows that Questions 1, 4, 5, 8, and 9 after analysis were classified as Factor 1: accessibility; Questions 2 and 3 were classified as Factor 2: usability; Questions 6, 7, and 10 were classified as Factor 3: satisfaction; and Question 11 was classified as Factor 4: learning.

\section{Qualitative analysis of behavioral observation}

This study introduces the nostalgic experience of science

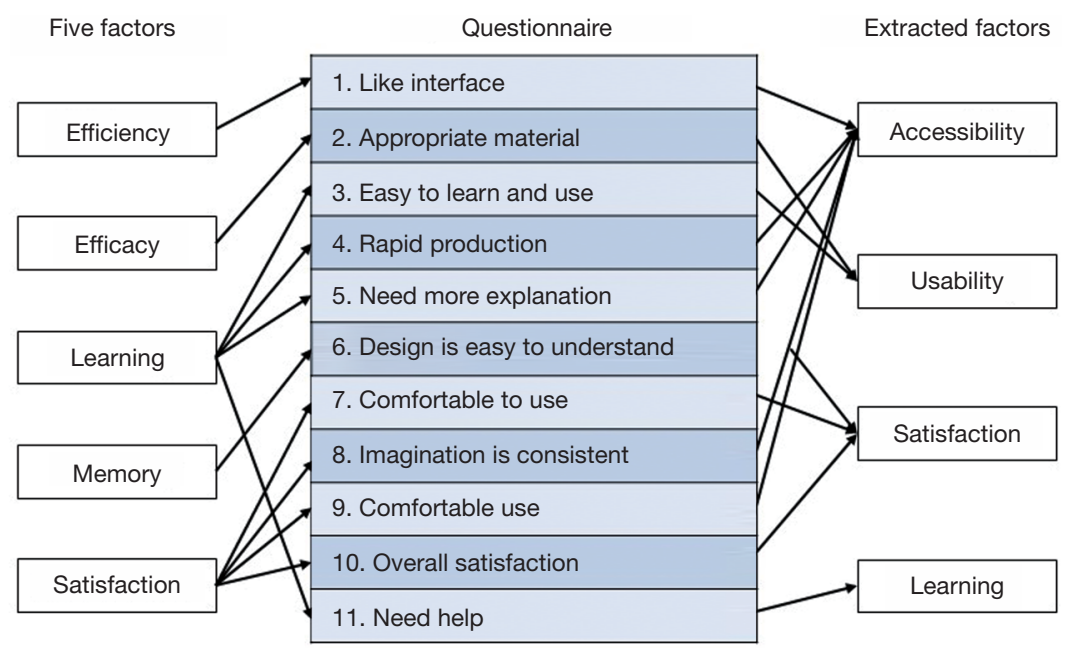

Figure 2 Comparison of the questionnaire structure and extracted factors. 
and technology. The subjects, with mild dementia, have the ability of experience the development of the "recall" storytelling system. The researchers have to observe the using behavior and emotional response of subject's experience. At the beginning, subject B1 and B2 were slightly excluded from using technology, but they were willing to try it through the guidance of researchers. Subject B3 always is smiling during process; Subject B5, B10, B17 have a lower willingness to use technology; Subject B6, B7, B8, B11, B13, B14, B16, B15, $\mathrm{B} 16, \mathrm{~B} 17$ are very positive in use and have a good technical experience; Subject B1-B8 performed well on this system, but subject B9 and B19 were more likely to be fatigued and unable to focus on activities for a long time.

\section{Sensory experience of technological nostalgic treatment}

(I) Overall, user feel good on using the system; B8 stated what one has done before; B8, B10, B12, B16, B18, B19 said that this system provides a good nostalgic feeling. It was generally considered the picture style look good; B6 says it was well designed;

(II) As for the auditory, most participants were familiar with the nostalgic background music;

(III) The user felt very reminiscence for the old photos presented in digital form. B6 and B8 felt that they had been returned to the former feelings; B4 and B13 thought of some things that were recalled in the past; B10 felt very happy, especially when they saw the food when they ate in young.

Sensory stimuli can directly observe the reaction of user. Through different visual experiences in the past, the presented past visual impressions give the dementia patients a new visual experience.

\section{Emotional experience of technological nostalgic treatment}

(I) Most of dementia patients have experience on using the technology products, and are interesting on operating the system we designed. B6 said that this system allows him to keep up with the times, it is not difficult to use a computer;

(II) By the screen, user can hear their own story and observe their own photos. B3, B6, B8, B12, B13, and B17 felt touching;

(III) By the nostalgic system, B1 recalls the past life situation was very happy; B3 was happy when he mentioned his granddaughter; B5 sometimes got angry and sometimes happy when he recall something; B6 felt that the life was a lot worse than it is; B7, B13, B17 stated the previous hard work days, no entertainment, and the situation is much better and satisfier than it was;

(IV) The nostalgic situation causes the curiosity of B6, B8, B11, B12, B18; B13 emphasized that it is interesting when operated first time; B1 and B5 had less curiosity about the system.

Technology can establish some new links through familiar things. Most people with dementia are not afraid of repelled by this nostalgic technology experience. Therefore, their emotional experience can touch the inner memory (one of implicit memory) of people with dementia.

\section{Action experience of nostalgic treatment of technology}

(I) The digital photo albums recalled the previous memories of the subjects. All subjects were associated with past related events, such as performing the movements of the fish and the process of transplanting;

(II) $\mathrm{B} 1, \mathrm{~B} 6, \mathrm{~B} 7$, and $\mathrm{B} 8$ will want to continue to record their further stories and leave good memories in digital;

(III) Most of user were willing to share their stories with their family or others. B6, B12, and B13 actively said that they would tell the children what they were; B17 could not share because their children were busy;

(IV) B6, B7, B8, and B18 will be happy if use other similar technology products in the future.

When people with dementia have a good technology experience and feelings, they will be willing to share the experience process; and with a good experience of technology, will be willing to try new products.

\section{Suggestions for behavior observations of persons with dementia}

During the experiment, caregivers accompanied the patient with dementia to operate the digital reminiscence system. It is found the subjects' finger are insufficient strength and flexibility, some of them cannot complete various movements, such as shaking hands, insufficient pressing strength, or inability to press for a long time during the operation. The touch operation interface should be simplified. Although the edited pictures and sound files can be saved, the subjects preferred real objects. if the story pictures can be output to present the story contents in revision, the subjects would own actual mementos.

\section{Conclusions}

With the advent of an aged society, the number of patients 
with dementia is increasing around the world. The family's care function is gradually declining with the low birth rate, causing the loading of family care is heavier. That would result in problems of social and economic. At present, the development of assistive technologies for the patients with dementia in Taiwan is mostly based on cognitive function training, while few systems combine reminiscence therapy features and joint caregivers with patients with dementia.

In this study, people with mild and moderate dementia in Changhua city were taken as the research subjects. Based on the investigation of reminiscence images in Taiwan, and by referring to the activities designed by care institutions for patients with dementia, a prototype of a digital reminiscence application system was developed and designed to facilitate caregivers accompanying patients with dementia for reminiscence Therapy. The system collects reminiscence elements and accesses old photos through digitalization, and combines both of "photo album" and "voice recordings", and "cognitive stimulation" to provide care providers with a platform to lead patients with dementia to carry out reminiscence Therapy. The digitalization system is helpful to record the operating data and preserve materials. The contribution and suggestions of this study are, as follows:

(I) The application system of digital technology is combined with reminiscence therapy. In the past, reminiscence therapy was mostly carried out by the caregivers who led patients with dementia to operate physical props. Regarding the expression changes of the subjects during the reminiscence process, the oral content was not completely recorded. Therefore, this study introduced digital technology into the reminiscence process, and built a digital reminiscence system to record the user's basic data and past experiences at the back end of the platform, in addition to facilitating management.

(II) The digital reminiscence system combines photo albums and audio recordings to enable families to completely preserve the stories of patients with dementia. That would improve the maintain of memory from traditional method that the paper photos tend to turn yellow, blue, or lose clarity with the passage of time. Stories told by the elderly are often neglected and forgotten, and it is only later that they regret for not keeping more memories. Almost of the auxiliary technologies developed for patients with dementia in Taiwan now focus on cognitive training, few systems are developed that combine digital storytelling with reminiscence therapy. Thus, this study not only provides cognitive stimulation to the different reminiscence materials of patients with dementia, it also provides families with a platform for storing stories and more memories can be preserved.

(III) Discussing the usability research of the digital reminiscence system for patients with dementia. Although there have been many related researches on the usability of the system in the past, there were still some deficiencies in the usability research from the perspective of patients with dementia. This study proposes four factors that affect the usability of the digital reminiscence application system: accessibility, usability, satisfaction, and learning, in order to provide reference for future research.

\section{Acknowledgments}

The authors would like to thank the Changhua Christian Hospital for supporting this research. This study gives special thanks to the experts, participants, and research team; without them this study could not have been completed. The authors also gratefully acknowledge the editor and anonymous reviewers for their valuable comments and constructive suggestions.

Funding: This research was based on work supported by the Ministry of Science and Technology of Taiwan, under contract MOST 107-2410-H-018-021.

\section{Footnote}

Conflicts of Interest: All authors have completed the ICMJE uniform disclosure form (available at http://dx.doi. org/10.21037/ht.2019.10.01). The authors have no conflicts of interest to declare.

Ethical Statement: The authors are accountable for all aspects of the work in ensuring that questions related to the accuracy or integrity of any part of the work are appropriately investigated and resolved. The study was conducted in accordance with the Declaration of Helsinki (as revised in 2013). The study was approved by the Research Ethics Committee of National Changhua University of Education, ethics board of no. NCUEREC-107-038. In accordance with this approval and informed consent was taken from all the participants.

Open Access Statement: This is an Open Access article distributed in accordance with the Creative Commons 
Attribution-NonCommercial-NoDerivs 4.0 International License (CC BY-NC-ND 4.0), which permits the noncommercial replication and distribution of the article with the strict proviso that no changes or edits are made and the original work is properly cited (including links to both the formal publication through the relevant DOI and the license). See: https://creativecommons.org/licenses/by-nc-nd/4.0/.

\section{References}

1. DeVaul RW, Pentland A, Corey VR. The memory glasses: subliminal vs. overt memory support with imperfect information. Seventh IEEE International Symposium on Wearable Computers, 2003. Proceedings; White Plains, NY, USA; 21-23 Oct, 2003:146-53.

2. Spector A, Woods B, Orrell M. Cognitive stimulation for the treatment of Alzheimer's disease. Expert Rev Neurother 2008;8:751-7.

3. Woods B, Aguirre E, Spector AE, et al. Cognitive stimulation to improve cognitive functioning in people with dementia. Cochrane Database Syst Rev 2012;(2):CD005562.

4. McDougall GJ, Buxen CE, Suen LJ. The process and outcome of life review psychotherapy with depressed homebound older adults. Nurs Res 1997;46:277-83.

5. Watt LM, Cappeliez P. Integrative and instrumental reminiscence therapies for depression in older adults: Intervention strategies and treatment effectiveness. Aging Ment Health 2000;4:166-77.

6. Astell A, Ellis MP, Alm N, et al. Stimulating people with dementia to reminisce using personal and generic photographs. International Journal of Computers in Healthcare 2010;1:177-98.

7. Guillemot S, Urien B. Legacy Writing among the Elderly: Conceptual Bases, Dimensioning and a Proposed Scale for Measuring Motivations. Recherche et Applications En Marketing (English Edition), 2010;25:25-43.

8. Alaoui M, Lewkowicz M. A living lab approach to involve elderly in the design of smart TV applications offering communication services. In: Ozok A, Zaphiris P editors. Online Communities and Social Computing. Lecture Notes in Computer Science, 8029. Berlin, Heidelberg: Springer, 2013:325-34.

9. Savulich G, Piercy T, Fox C, et al. Cognitive Training Using a Novel Memory Game on an iPad in Patients with Amnestic Mild Cognitive Impairment (aMCI). Int J Neuropsychopharmacol 2017;20:624-33.

10. Asghar I, Cang S, Yu H. Usability evaluation of assistive technologies through qualitative research focusing on people with mild dementia. Comput Human Behav 2018;79:192-201.

11. Boman IL, Lundberg S, Starkhammar S, et al. Exploring the usability of a videophone mock-up for persons with dementia and their significant others. BMC Geriatr 2014;14:49.

12. Tchalla AE, Lachal F, Cardinaud N, et al. Efficacy of simple home-based technologies combined with a monitoring assistive center in decreasing falls in a frail elderly population (results of the Esoppe study). Arch Gerontol Geriatr 2012;55:683-9.

13. Zhang S, McClean SI, Nugent CD, et al. A predictive model for assistive technology adoption for people with dementia. IEEE J Biomed Health Inform 2014;18:375-83.

14. Pecorini BC, Duplaa E. Narrative Gerontology and Digital Storytelling: What Benefits for Elders? MOJ Public Health 2017;6:00192.

15. Lazar A, Thompson H, Demiris G. A systematic review of the use of technology for reminiscence therapy. Health Educ Behav 2014;41:51S-61S.

16. Lin YC, Dai YT, Hwang SL. The effect of reminiscence on the elderly population: a systematic review. Public Health Nurs 2003;20:297-306.

17. Cotelli M, Manenti R, Zanetti O. Reminiscence therapy in dementia: a review. Maturitas 2012;72:203-5.

18. Subramaniam P, Woods B. The impact of individual reminiscence therapy for people with dementia: systematic review. Expert Rev Neurother 2012;12:545-55.

19. Woods B, Spector A, Jones C, et al. Reminiscence therapy for dementia. Cochrane Database Syst Rev 2005;(2):CD001120.

20. Subramaniam P, Woods B. Towards the therapeutic use of information and communication technology in reminiscence work for people with dementia: a systematic review. International Journal of Computers in Healthcare 2010;1:106-25.

21. Preece J, Rogers Y, Sharp H, Interaction design: Beyond human-computer interaction. 1st edition. USA: John Wiley \& Sons, Inc., 2002.

doi: 10.21037/ht.2019.10.01

Cite this article as: Wu PF, Wang WF, Fan KY, Hsu YZ, Huang CW. Research on the design and usability of Taiwan's native digital reminiscence therapy application system for patients with dementia. Health Technol 2019;3:9. 